\title{
Electrostatic topology of ferroelectric domains in $\mathrm{YMnO}_{3}$
}

\author{
Tobias Jungk ${ }^{1}$, Ákos Hoffmann ${ }^{1}$, Manfred Fiebig ${ }^{2}$, and Elisabeth Soergel ${ }^{1 *}$ \\ ${ }^{1}$ PI, Universität Bonn, Wegelerstraße 8, 53115 Bonn, Germany and \\ ${ }^{2}$ HISKP, Universität Bonn, Nussallee 14-16, 53115 Bonn, Germany
}

\begin{abstract}
Trimerization-polarization domains in ferroelectric hexagonal $\mathrm{YMnO}_{3}$ were resolved in all three spatial dimensions by piezoresponse force microscopy. Their topology is dominated by electrostatic effects with a range of 100 unit cells and reflects the unusual electrostatic origin of the spontaneous polarization. The response of the domains to locally applied electric fields explains difficulties in transferring $\mathrm{YMnO}_{3}$ into a single-domain state. Our results demonstrate that the wealth of non-displacive mechanisms driving ferroelectricity that emerged from the research on multiferroics are a rich source of alternative types of domains and domain-switching phenomena.

PACS numbers:
\end{abstract}


In materials with a coexistence of magnetic and ferroelectric order, called multiferroics,,$-\underline{-3}$ the most prominent type of ferroelectricity, i.e., ferroelectricity of the displacive type found in perovskites like $\mathrm{BaTiO}_{3}$, is usually avoided. $\stackrel{4}{-}$ Therefore, multiferroics research lead to the awareness of a wealth of alternative mechanisms driving the emergence of a spontaneous polarization. This includes ferroelectricity from electron lone pairs, charge order, helical spin structures, electrostatic effects, and more. ${ }^{2.5}$ The unconventional origin of the spontaneous polarization in these systems should also affect the basic properties of a ferroelectric such as the distribution and switching behavior of its domains. However, in spite of the implications of this aspect for technological applications it has not attracted much attention thus far.

Very recently, the ferroelectric domain structure of hexagonal $\mathrm{YMnO}_{3}$ was investigated. $\underline{6}$ Hexagonal manganites are textbook multiferroics in which the spontaneous polarization is induced by electrostatic instead of displacive effects. Choi et al. found that perpendicular to the spontaneous polarization intriguing intersections of as many as six ferroelectric domains are common to $\mathrm{YMnO}_{3}$ and related this to the atomic displacement at the domain wall. In this letter we show that in addition (or alternatively) the unusual domain topology is a direct consequence of the electrostatic nature of the ferroelectric state in $\mathrm{YMnO}_{3}$. This is derived from piezoresponse force microscopy (PFM) measurements showing that in spite of the anisotropic crystal structure kaleidoscopic intersections of domain walls are present in all three spatial dimensions. At the intersections electrostatic repulsion leads to a relative displacement of polarization and trimerization domains that are otherwise rigidly coupled. PFM tip-poling experiments confirm that the electrostatic discontinuity at the domain walls controls the spontaneous polarization within a range of about 100 unit cells.

In hexagonal $\mathrm{YMnO}_{3}$ the $\mathrm{Mn}^{3+}$ ions are found in a rare fivefold coordination with the $\mathrm{O}^{2-}$ ions. Planes of $\mathrm{MnO}_{5}^{3+}$ bipyramids are interspaced with planes of $\mathrm{Y}^{3+}$ ions along the hexagonal $z$ axis. Ferroelectricity emerges in two steps. ${ }^{7.8}$ At $1270 \mathrm{~K}$ tilting of the $\mathrm{MnO}_{5}^{3+}$ polyhedra and corrugation of the $\mathrm{Y}^{3+}$ layers occurs. At $920 \mathrm{~K}$ additional displacement of the $\mathrm{MnO}_{5}^{3+}$ polyhedra induces a spontaneous polarization $P_{z}$ with a saturation value of $5.6 \mu \mathrm{C} / \mathrm{cm}^{2}$. The transitions are driven by electrostatic and geometric effects, rather than by the usual changes in chemical bonding associated with displacive ferroelectric phase transitions as in perovskite oxides. This mechanism permits the coexistence of magnetism and ferroelectricity so that the compound becomes multiferroic at cryogenic temperatures. $\frac{5}{-}$ As shown in Fig. 1 unit-cell tripling at $1270 \mathrm{~K}$ leads to three trimerization domains $(\alpha, \beta, \gamma)$. The polarization along $+z$ or $-z$ emerging at $920 \mathrm{~K}$ leads to a 
total of six domains $\left(\alpha^{ \pm}, \beta^{ \pm}, \gamma^{ \pm}\right)$. The trimerization domains are translation domains for which the identification as $\alpha, \beta$, or $\gamma$ is ambiguous. In contrast, the polarization domains are $180^{\circ}$ domains with a unique assignment of + and - . From a variety of experiments as-grown ferroelectric domains in $\mathrm{YMnO}_{3}$ are known to possess an extension of $\lesssim 1 \mu \mathrm{m} . \underline{9-12}^{-12}$ but a detailed study of their topology has only been presented by Choi et al.

$\mathrm{YMnO}_{3}$ samples were flux-grown $z$-oriented platelets with a lateral extension of a few mm and a thickness in the order of $100 \mu \mathrm{m} .^{13}$ Chemical-mechanical polishing with a silica slurry was applied to the $z$ face and to $x$ - and $y$-oriented bars cut from one platelet. The distribution of ferroelectric domains was measured by PFM which allows us to probe all three crystallographic directions non-invasively with an impressive sensitivity and high spatial resolution. ${ }^{14,15}$

Figure 2 shows the $x, y$, and $z$ faces of as-grown $\mathrm{YMnO}_{3}$ crystals from the same batch under ambient conditions. As expected, all faces reveal domains of $\lesssim 1 \mu \mathrm{m}$ with two grey levels corresponding to domains with $+P_{z}$ (bright) or $-P_{z}$ (dark). However, the topology of the ferroelectric domains is striking. On the $z$ face of the crystal the same kaleidoscopic domain structure with meeting points of six domains as in Ref. $\underline{6}$ is obtained. In Ref. $\underline{6}$ the intersections were assigned to $\alpha^{+}, \beta^{-}, \gamma^{+}, \alpha^{-}, \beta^{+}, \gamma^{-}$(anti-) vortices of domains with a rigid clamping of trimerization and polarization domain walls. The clamping was attributed to the microscopic structure at the domain wall: The coexistence of trimerization and polarization walls minimizes the displacement of the $\mathrm{Y}^{3+}$ position with respect to the paraelectric phase which might be energetically favorable. This argument referred to domain walls in the $x y$ plane so that kaleidoscopic intersections are expected in this plane only. However, Fig. 2 reveals that in spite of the highly anisotropic uniaxial structure of the $\mathrm{YMnO}_{3}$ crystal the distribution of the domains is almost isotropic with only a slight elongation along $z$. In all three spatial dimensions the peculiar ferroelectric domain topology with meeting points of six domains is observed. We therefore conclude that in addition (or alternative) to mechanisms rooting in the microscopic structure of the $\mathrm{YMnO}_{3}$ unit cell a more general mechanism that is less sensitive to the microscopic anisotropy must be responsible for the domain structure in Fig. 2,

For elucidating this issue we conducted high-resolution PFM scans of the domain vortex region. The images in Fig. 3 reveal that the six trimerization-polarization domains do not really meet in one point. In the majority of cases three equally polarized domains approach one another up to a distance of about $30 \mathrm{~nm}$ on the $z$ face and of about $100 \mathrm{~nm}$ on the $x$ and $y$ faces. Hence, at the center of the corresponding domain vortex the spontaneous polarization is $+P_{z}$ or $-P_{z}$ instead of 
approaching zero. In a minority of cases two domains of equal polarization are connected via a thin bridge but separated from the third, equally polarized domain. On the $x$ and $y$ faces this scenario is met an order of magnitude less often than the former one. For the $z$ face the occurrence of the second scenario is not clear. As in the case of Fig. 2 the local microscopy of the domain walls cannot be responsible for the topology of domains observed here. Domain walls in ferroelectrics are at best a few unit cells wide while separations in the order of 100 unit cells are observed in Fig. 3. This rather indicates that Coulomb interactions determine the domain structure at the vortex. The Coulomb force is a central force, thus acting in all three spatial dimensions and it is a long-range force that can act across many unit cells.

In order to understand the relation of the trimerization and polarization domain walls to the electrostatic repulsion and vortex structure of the domains we subjected the $\mathrm{YMnO}_{3}$ crystal in Fig. 4 to an electric poling field. A DC voltage of $50 \mathrm{~V}$ was applied via the SFM tip along the $z$ axis while scanning a square of $5 \times 5 \mu \mathrm{m}^{2}$. In contrast to Ref. 6 the voltage was applied noninvasively and the same region was imaged before and after poling. For the domain vortex we have to distinguish between the two scenarios depicted in Figs. 4(a) and 4(b): (1) an $\alpha^{+}, \alpha^{-}, \beta^{+}$, $\beta^{-}, \gamma^{+}, \gamma^{-}$and (2) an $\alpha^{+}, \beta^{-}, \gamma^{+}, \alpha^{-}, \beta^{+}, \gamma^{-}$sequence of domains. 16 Scenario (1) involves two types of boundaries: polarization-trimerization and polarization-only walls. This may occur if the trimerization walls are so stable that each trimerization domain splits into two polarization domains. Scenario (2) involves polarization-trimerization walls only. This will occur if the creation of additional trimerization domains costs less energy than the creation of a polarization-only wall. Scenario (2) was proposed in Ref. 6 but not explicitly confirmed there because trimerization and polarization domains were observed in separate experiments, i.e., by force and electron microscopy, respectively.

According to the scan shown in Fig. 4(d) all walls respond to the electric field, thus indicating that scenario (2) as depicted in Fig. 4(b) holds. This result was confirmed by annealing experiments in which a sample was heated to $1150 \mathrm{~K}$ for three hours. After re-cooling all the domain walls, including the trimerization walls, were found to have moved. We therefore conclude that in $\mathrm{YMnO}_{3}$ trimerization and polarization walls are rigidly coupled except in the vortex region where the correlation becomes "flexible" with a relative spatial displacement of trimerization and polarization walls as shown in Fig. 3(c).

Note that in contrast to displacive ferroelectrics it is not possible to convert the entire $\mathrm{YMnO}_{3}$ sample into a single-domain state. Figure 4(d) shows that the presence of remanent interstitial 
domains polarized oppositely to the applied electric field are enforced by the electrostatic discontinuity at the domain wall. This nicely reflects the electrostatic nature of the ferroelectric state and explains why in former experiments evidence for a multi-domain state was still obtained when the coercive field was exceeded by an order of magnitude. ${ }^{12}$ Quantitative analysis of Fig. 4(d) reveals a width of $60 \pm 10 \mathrm{~nm}$ for the interstitial domain which matches the electrostatic reach found in Fig. 3.

Our observations thus reveal the following scenario. Growth-induced trimerization leads to intersections where one $\alpha$, one $\beta$, and one $\gamma$ domain meet. The electrostatic discontinuity associated to a trimerization wall controls the ferroelectric polarization up to a distance of about 100 unit cells. It enforces a reversal of polarization whenever a trimerization wall is crossed. The simplest arrangement of domains satisfying these requirements involve kaleidoscopic intersection of six domains as shown in Fig. 4(b) and Ref. 6. At the intersection the electrostatic repulsion between equally polarized domains is solved by a decoupling of trimerization and polarization domains near the center of the domain vortex.

In summary the unusual kaleidoscopic topology of ferroelectric trimerization-polarization domains in multiferroic hexagonal $\mathrm{YMnO}_{3}$ was shown to be a consequence of electrostatic effects which reflects the unusual electrostatic origin of the spontaneous polarization in this compound. The discontinuity at the domain walls leads to locally polarizing fields that determine the spontaneous polarization within a range of 100 unit cells in all three spatial dimensions. At the intersection of domains this leads to a flexible coupling of trimerization and polarization domains with correlated, but non-overlapping domain walls. Away from the intersection trimerization and polarization walls coincide with domains maintaining a width of at least 100 unit cells. Even beyond saturation fields a remanent ferroelectric multi-domain structure is inherent to the sample which explains former difficulties to transform $\mathrm{YMnO}_{3}$ into a single-domain state. Our results show that the wealth of non-displacive mechanisms driving ferroelectricity that emerged from the present intense research activities on multiferroics are a potential source of alternative domain and polarization phenomena with basic-research as well as technological implications.

The authors thank the Deutsche Telekom AG and the DFG for financial support and J. F. Scott for useful advice about multidimensional order parameters.

* Electronic address: soergel@uni-bonn.de 
1 W. Eerenstein, N. D. Mathur, and J. F. Scott, Nature 442, 759 (2006).

2 D. Khomskii, Physics 2, 20 (2009).

3 M. Fiebig, J. Phys. D 38, 123 (2005).

4 N. A. Hill, J. Phys. Chem. B 104, 6694 (2000).

5 B. B. van Aken, T. T. M. Palstra, A. Filippetti, and N. A. Spaldin, Nat. Mater. 3, 164 (2004).

6 T. Choi, Y. Horibe, H. T. Yi, Y. J. Choi, W. Wu, and S.-W. Cheong, Nat. Mater. 9, 253 (2010); M. Mostovoy, Nat. Mater. 9, 188 (2010).

7 T. Lonkai, D. G. Tomuta, U. Amann, J. Ihringer, R. W. A. Hendrikx, D. M. Többens, and J. A. Mydosh, Phys. Rev. B 69, 134108 (2004).

8 C. J. Fennie and K. M. Rabe, Phys. Rev. B 72, 100103(R) (2005).

9 M. Šafránková, J. Fousek, and S. A. Kižaev, Czech. J. Phys. B 17, 559 (1967).

10 A. S. Oleinik and V. A. Bokov, Sov. Phys. Solid State 17, 560 (1975).

11 C. C. Neacsu, B. B. van Aken, M. Fiebig, and M. B. Raschke, Phys. Rev. B 79, 100107(R) (2009).

12 M. Fiebig, D. Fröhlich, Th. Lottermoser, and M. Maat, Phys. Rev. B 66, 144102 (2002).

13 S. H. Kim, S. H. Lee, T. H. Kim, T. Zyung, Y. H. Jeong, and M. S. Jang, Cryst. Res. Technol. 35, 19 (2000).

14 T. Jungk, Á. Hoffmann, and E. Soergel, New J. Phys. 10, 013019 (2008).

15 T. Jungk, Á. Hoffmann, and E. Soergel, New. J. Phys. 11, 033029 (2009).

16 Scenarios like (1) $\alpha^{-}, \alpha^{+}, \beta^{-}, \beta^{+}, \gamma^{-}, \gamma^{+}$or (2) $\alpha^{+}, \gamma^{-}, \beta^{+}, \alpha^{-}, \gamma^{+}, \beta^{-}$are equivalent. 


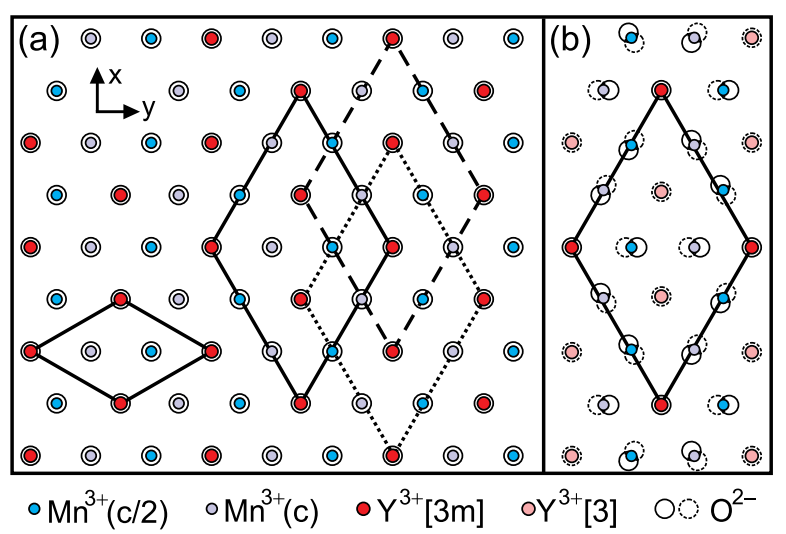

FIG. 1: (Color) (a) $\mathrm{YMnO}_{3}$ crystal at $>1270 \mathrm{~K}$ with the unit cell (small diamond) and three choices for the trimerization at $1270 \mathrm{~K}$ (large diamonds). (b) Ferroelectric crystal with tripled unit cell. Legend $-\mathrm{Mn}^{3+}$ ions: $z$ position in the unit cell; $\mathrm{Y}^{3+}$ ions: local symmetry. 

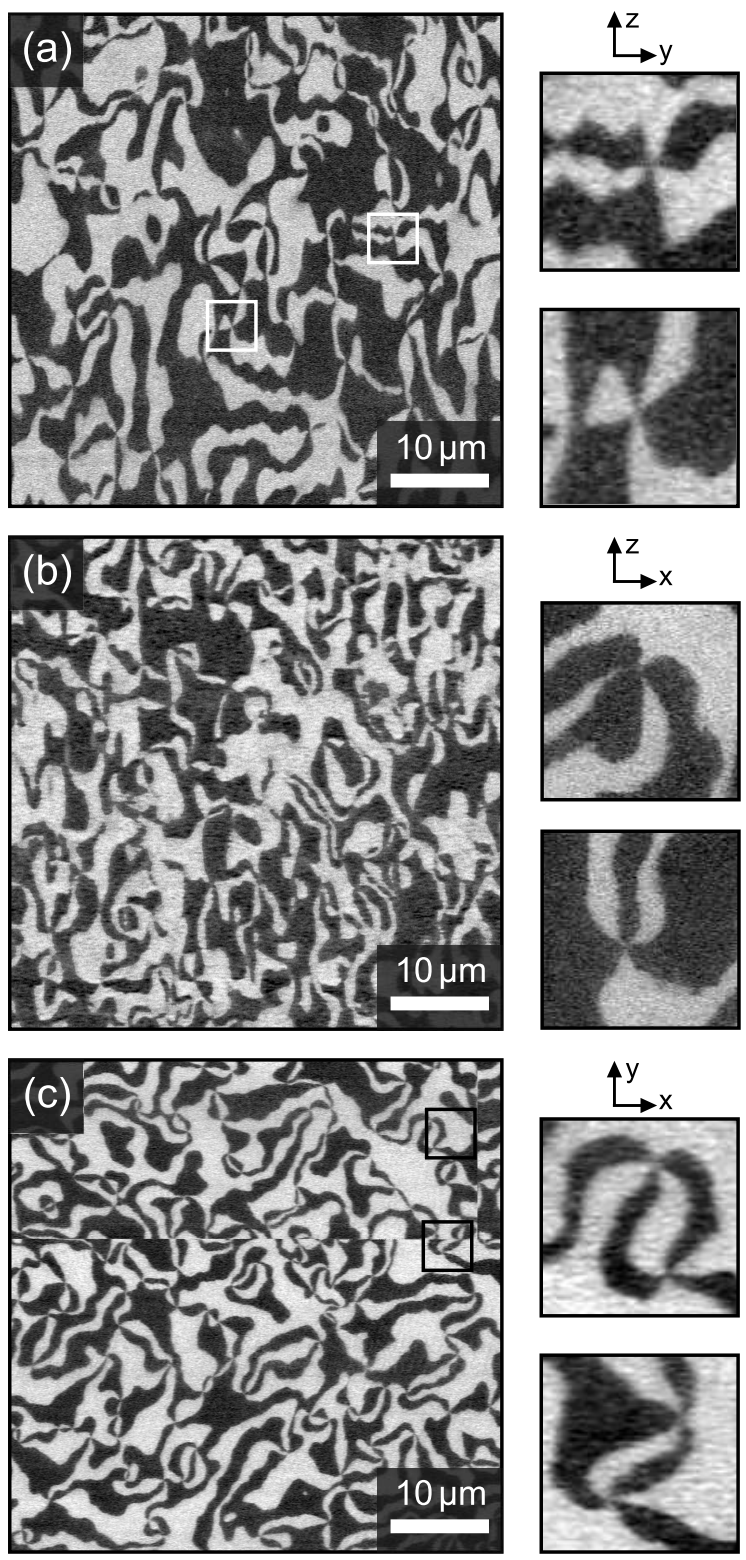

FIG. 2: PFM images $\left(50 \times 50 \mu \mathrm{m}^{2}\right)$ of the $x, y$ and $z$ faces of as-grown $\mathrm{YMnO}_{3}$ crystals from the same batch. Bright and dark areas correspond to ferroelectric domains with $+P_{z}$ and $-P_{z}$, respectively. 


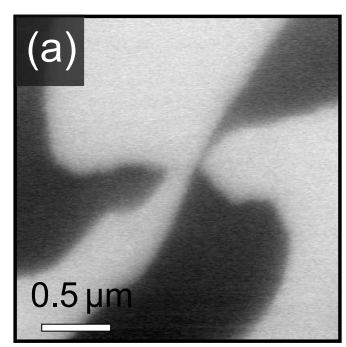

$y$-face

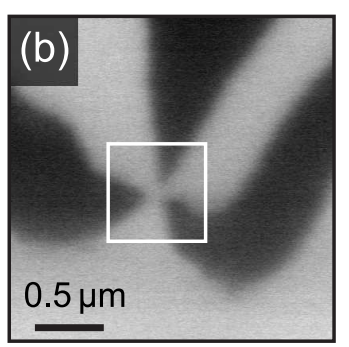

$x$-face

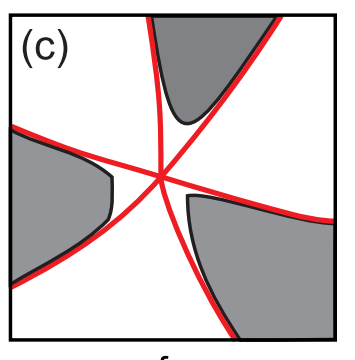

$x$-face

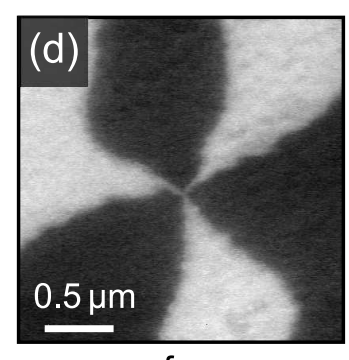

z-face

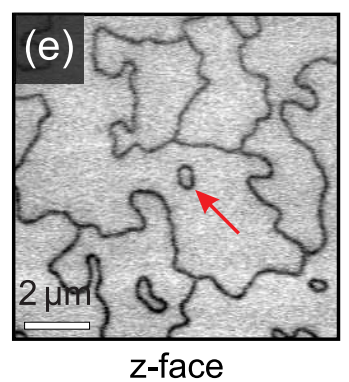

FIG. 3: (Color) High-resolution PFM images of the trimerization-polarization domains. (a, b, d) Exemplary images of the $y, x$, and $z$ face as discussed in the text. (c) Sketch of the domain structure in (b) showing trimerization and polarization walls as straight red lines and boundaries of grey areas, respectively. (e) Near-single $+P_{z}$ domain with $-P_{z}$ domains shrunk to stripes of $\sim 60 \mathrm{~nm}$. Note that closed-loop domains (arrow) are also observed. 
(a)
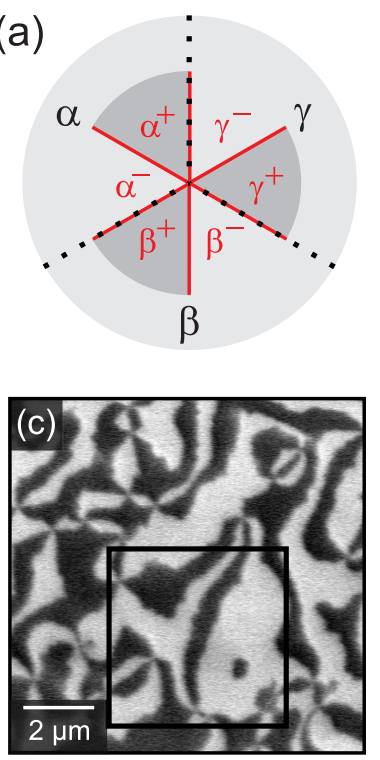

(b)
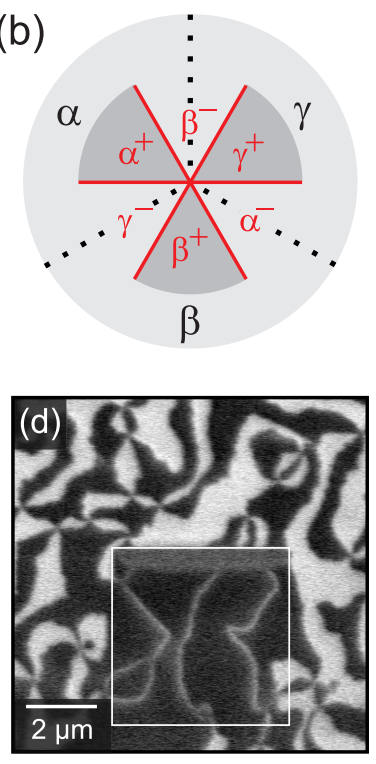

FIG. 4: (Color) Structure of trimerization and polarization domains and their response to an electric field. (a, b) Scenarios 1 and 2 as discussed in the text. $\alpha, \beta, \gamma$ and dotted black lines denote the distribution of domains in the trimerized phase at $>920 \mathrm{~K} . \alpha^{ \pm}, \beta^{ \pm}, \gamma^{ \pm}$and straight red lines denote the distribution of domains in the ferroelectric phase at $<920 \mathrm{~K}$. (c, d) PFM image $\left(10 \times 10 \mu \mathrm{m}^{2}\right)$ of a $z$ faced $\mathrm{YMnO}_{3}$ crystal (c) before and (d) after tip-voltage poling. 